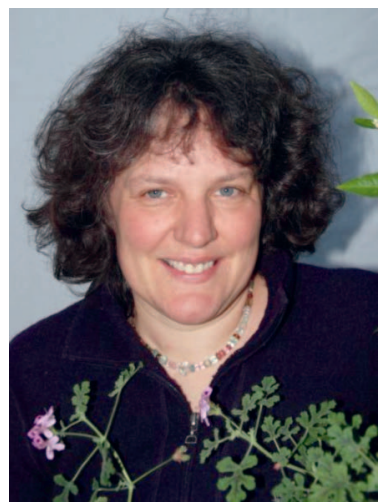

Frau Dal Cero, wie schätzen Sie die derzeitige Situation und die $\mathrm{Zu}$ kunft der Komplementärmedizin in der Schweiz ein?

Komplementärmedizin ist ein unverzichtbarer und in der Bevölkerung gut verankerter Bestandteil der medizinischen Landschaft in der Schweiz. Als Nutzerin des komplementärmedizinischen Angebotes schätze ich die Möglichkeit, je nach Bedürfnis und Fragestellung ein vielfältiges und qualitativ hochstehendes Angebot zur Verfügung zu haben - allerdings ein so buntes und vielfältiges Angebot, dass man auch rasch den Überblick darin verlieren kann.

Der Dialog zwischen komplementärmedizinischen Richtungen und konventionellen Ansätzen scheint mir für die Zukunft wegweisend, wie er beispielsweise in der Integrativen Medizin bereits gepflegt wird.

Wichtig bleibt meines Erachtens für die Komplementärmedizin, dass auch in Zukunft Spielräume und Chancen kreativ genutzt werden - mit einem soliden Verständnis für Qualität. Ein Beispiel für eine gelungene Entwicklung aus der Pflege ist etwa die vermehrte Anwendung von Wickeln und Kompressen, die sich aufgrund der Initiative einer engagierten Gruppe von Pflegefachfrauen in den letzten Jahren in verschiedenen Institutionen etabliert hat.

\title{
Kräuterkundige in der Schweiz
}

Maja Dal Cero, dipl. Natw. ETH, beschäftigt sich im Rahmen der Ethnobotanik und Ethnomedizin mit der Vielfalt der Medizinalpflanzen und -kräuter in der Schweiz. Sie hat ein Studium der Umweltnaturwissenschaften an der ETH Zürich absolviert und eine Ausbildung in Pflanzenheilkunde und Aromatherapie abgeschlossen. Momentan arbeitet Frau Dal Cero an ihrer Dissertation in der Gruppe von Dr. Caroline Weckerle, Institut für Systemische Botanik der Universität Zürich, zum Thema «Kräuterkundige in der Schweiz» und richtet einen Botanikzirkel für Heilpflanzenpraktikerinnen und -praktiker in Therapie und Pflege in Schaffhausen und Umgebung aus.

Sie sind unter anderem im Bereich der Ethnobotanik und Phytotherapie tätig. Mit welchen Forschungsbereichen beschäftigen Sie sich genau - vor allem in Bezug auf die Schweiz?

Zurzeit sind es kräuterkundige Menschen in der Schweiz, die mich im Rahmen einer ethnobotanischen Studie am Institut für Systematische Botanik der Universität Zürich intensiv beschäftigen. Welche Arzneipflanzen, einheimische oder exotische, bevorzugen sie? Woher haben sie ihr Wissen und wie geben sie es weiter? Mit welchen Vorstellungen über Wirkungsweisen von Arzneipflanzen oder über Krankheit und Gesundheit arbeiten Kräuterkundige gegenwärtig? Anhand von Experteninterviews sowie Fragebogen-Erhebungen werden diese Fragestellungen bearbeitet. Der Reiz an der ethnobotanischen Forschung in der Schweiz liegt für mich im Eruieren der Beziehungen zwischen Menschen und (Arznei-)Pflanzen in einem komplexen, von wirtschaftlichen und regulatorischen Bestimmungen geprägten Umfeld.

Wie es sich nun nach rund 50 Experteninterviews zeigt, können Arzneipflanzen im oben angesprochenen Dialog der verschiedenen medizinischen Kulturen eine zentrale Rolle einnehmen, da sie sowohl in der naturwissenschaftlich orientierten Phytotherapie als auch in verschiedenen komplementärmedizinischen Modellen eine wichtige Rolle spielen.

Sie haben bereits die Bücher «Unsere Heilpflanzen» und "Pflanzen für die Gesundheit - Botanik in der Praxis» veröffentlicht. Welche Aspekte sprechen Sie insbesondere an? Sind weitere Veröffentlichungen in Planung?

Pflanzen sind in erster Linie Lebewesen, denen wir auf Schritt und Tritt begegnen und von denen unser Leben im engsten Sinne abhängt - diese Sichtweise ist wohl geprägt durch mein Studium der Umweltnaturwissenschaften an der ETH Zürich, in dem ökologische Zusammenhänge im Zentrum standen. Gleichzeitig sprechen Pflanzen menschliche Sinne unmittelbar an, durch ihre Ästhetik, über ihre Düfte, ihren Geschmack, als Nahrungsmittel, als Textilien, als Baumaterialien oder eben als Arzneipflanzen.

Mir ist es deshalb ein besonderes Anliegen, Möglichkeiten für den direkten Kontakt mit den Pflanzen an ihren natürlichen Standorten $\mathrm{zu}$ schaffen. Beide Bücher vermitteln fundiertes Grundwissen und dienen als zuverlässige Begleiter für Begegnungen mit Arzneipflanzen in ihren natürlichen Lebensräumen.

Das erste Buch über «Arzneipflanzenbotanik» ist direkt aus meiner

\section{KARGER}

Fax +49761 4520714 
Tätigkeit als Dozentin für botanische Grundlagen entstanden. Es fehlte bislang ein Lehrmittel, das botanische Grundbegriffe, Systematik sowie ökologische und historische Zusammenhänge leicht verständlich darstellt für Menschen, die mit Arzneipflanzen arbeiten - in der Pflege oder als Therapeutinnen und Therapeuten.

«Unsere Heilpflanzen» ist die Weiterführung des gleichnamigen Werks von Prof. Dr. H. Flück, das eine Auswahl von 150 gut dokumentierten, in der Schweiz wachsenden Heilpflanzen in Wort und Bild präsentiert.

Nachdem zuerst die botanischen Grundlagen sowie Pflanzenporträts im Vordergrund standen, rücken aktuell die kräuterkundigen Menschen ins Zentrum. Geplant und nun langsam am Entstehen ist ein Werk über Kräuterkunde/Kräuterkundige in der Schweiz, das den Bogen von volksheilkundlichem Pflanzenwissen bis zur wissenschaftlich geprägten Phytotherapie spannt.

Was können Sie uns zum Botanikzirkel in Schaffhausen berichten? Gibt es darüber hinaus Kurse oder Fortbildungsangebote, an denen Sie mitwirken bzw. die Sie empfehlen möchten?

Der «Botanikzirkel» ist vor über 12 Jahren entstanden aus einer kleinen Gruppe Menschen, die beruflich mit Heilpflanzen arbeiten (Therapie, Pflege, Anbau, Lehre), aus dem Wunsch, die eigene Arbeit zu reflektieren und weiterzuentwickeln. Der Erfahrungsaustausch verlangt viel Eigeninitiative der Teilnehmenden; dafür gibt es eine individuell massgeschneiderte Weiterbildung. In der Zwischenzeit haben sich mehrere heterogen zusammengesetzte Gruppen gebildet, die jeweils ihre etwas eigene "Kultur» mit den spezifischen Interessen in Bezug auf Arzneipflanzen haben. Die regelmässigen Diskussionen mit den «Arzneipflanzenpraktikerinnen und -praktikern» aus den unterschiedlichsten Richtungen sind wiederum für meine eigene Arbeit bereichernd und inspirierend.

Es gibt heute ein breites Angebot an Pflanzenheilkunde-/Phytotherapie-Ausbildungen von privaten Schulen für nichtärztliche Therapeutinnen und Therapeuten sowie die von der FMH anerkannte Weiterbildung der Schweizerischen Medizinischen Gesellschaft für Phytotherapie (SMGP). Diese bietet ausserdem mit den botanischen Exkursionen eine gute $\mathrm{Ge}$ legenheit, die Arzneipflanzen nicht nur aus Büchern und als Arzneimittel zu kennen, sondern ihre Eigenheiten und bevorzugten Lebensbedingungen direkt zu erfahren.

Eine etwas andere Perspektive eröffnet der Weiterbildungsgang CAS «Ethnobotanik und Ethnomedizin» an der Universität Zürich, der in einem kompakten Programm den einmaligen Rahmen bietet, sich vertieft mit Fragen der (Arzneipflanzen-)Nutzung in einem globalen Kontext auseinanderzusetzen.

Welches komplementärmedizinische Heilmittel bzw. welche Heilpflanze fasziniert Sie persönlich am meisten? Warum?

Die Engelwurz - die aus dem hohen Norden stammende Erzengelwurz (Angelica archangelica) wie auch die kleinere einheimische Verwandte Waldengelwurz (Angelica sylvestris) faszinieren mich seit längerer Zeit. Jede Begegnung mit ihrer imposanten Erscheinung im Wald oder im Garten ist eindrücklich. Mittelalterliche Legenden und Geschichten, die sich um die Engelwurz ranken, berichten von ihrer unglaublichen Wirkung sogar gegen die Pest. In der Volksheilkunde ist Angelica - bekannt auch unter dem Namen Brustwurz - Bestandteil von Brust- und Hustensalben. Die mit Zucker eingekochten Stängel werden zu einer grasgrünen Delikatesse; verschiedene Klosterliköre führen die Engelwurz als wichtigste Ingredienz auf. Und nicht zuletzt lassen sich wohltönende Flöten aus den hohlen
Stängeln der Engelwurz schnitzen. In den Berggebieten tritt die botanisch verwandte Meisterwurz (Peucedanum ostruthium) oft an Stelle der Engelwurz.

Allein schon diese unvollständige Auflistung einzelner Aspekte der Engelwurz zeigt ihre grosse kulturgeschichtliche Bedeutung. In der Phytotherapie wird Engelwurz hauptsächlich als Amara aromatica zur Verdauungsunterstützung eingesetzt. Sie gehört damit unter anderen zu denjenigen Arzneipflanzen, die aufgrund einer nüchternen Wirkstoffbetrachtung in der modernen Phytotherapie eher ein Randdasein fristen. Aus ethnobotanischer Perspektive kann ein wesentlich grösseres Potenzial dieser Pflanzen ausgemacht werden, das sich vielleicht auch in Zukunft wieder entfalten kann.

Wie sind Sie ursprünglich mit der Komplementärmedizin in Berührung gekommen? Was interessiert Sie daran besonders?

Die Pflanzenwelt und das Experimentieren damit haben mich schon in Kindertagen fasziniert. Im praktischen Umgang mit Heilpflanzen in einem weiten Sinne lässt sich eine grosse Kreativität ausleben. Die breite Beschäftigung mit Arzneipflanzen, nicht nur als Wirkstoffoder Rohstofflieferanten, hat rasch dazu geführt, mich mit weiteren komplementärmedizinischen Modellen auseinanderzusetzen. Mich interessieren im Besonderen die unterschiedlichen Sichtweisen und Erklärungsansätze, die verschiedenen komplementärmedizinischen Richtungen zugrunde liegen. Verblüffend und bereichernd finde ich es immer wieder, festzustellen, wie mit sehr unterschiedlichen «Weltbildern» gute therapeutische Erfolge erzielt werden können.

Sehrgeehrte Frau Dal Cero, herzlichen Dank für das Interview!

Interview: Alexander Eitner 